\title{
Acker oder Weide? Die Zivilisation der Steppe und die Administration der Nomaden
}

\author{
Peasants or Pastures? The Civilization of the \\ Steppe and the Administration of Nomads
}

\author{
Olaf Günther
}

\begin{abstract}
The article is about the interactions between the Russian Empire and the nomadic tribes in the steppes of Central Asia. Nomadic economies and culture were well integrated into the surrounding environments but were urged by the Russian colonial administration to change to the sedentary lifestyle of an agrarian society. With this step Russian colonial politics hoped to solve the problems arising in its Central regions following the abolition of serfdom and the ensuing problem of scarcity of common land. The answer to these problems was contained in the sedentarisation of the nomads. This is why the Russian colonial administration oriented its general education of the nomads towards farming and agriculture. The aim was to introduce the advantages of agrarian economies through agrarian schooling. This experiment failed however, and the permanent settlement of the Steppes was only successful after the education of Russian settlers. The article follows the implementation of the school models through the debate on sedentarisation.
\end{abstract}

Keywords: Russland; Zentralasien; Kolonialismus; Nomadismus; Bildung

Das 19. Jahrhundert war für Russland das Jahrhundert, in dem es die europäische Bühne als Kolonialmacht betrat. Zwar hatten schon im 17. Jahrhundert russische Siedler und Kaufleute die Weiten Sibiriens erschlossen und von hier aus wirtschaftliche Kontakte mit China aufgenommen, geopolitisch war dies jedoch nicht von Belang. Das 19. Jahrhundert war die Epoche, in der das Imperium - noch nicht der Nationalstaat - die im Weltmaßstab dominante territoriale Organisationsform von Macht darstellte (Osterhammel 2008, 606). In den Jahren 1865 und 1867, als Russland die Steppengebiete Zentralasiens besetzte, die heute den Großteil des Staates Kasachstan bilden, legte es den Grundstein zu einem bedeutsamen historischen Konflikt des 19. Jahrhunderts, der heute als "Great Game“ (Hopkirk 1994) bekannt ist und in der Bildung Afghanistans als Pufferstaat zwischen dem „englischen Löwen“ und dem „russischen Bären“ gipfelte. Ein wichtiges Anliegen des Zaren Alexander II. (1855-1881) war, die Interessen der Zentralmacht in St. Petersburg stärker an der Peripherie durchzusetzen. Die Geschicke am Rande des Reiches wurden unter seinem Vater Nikolaus I. (1825-1855) vor allem den Wehrbauern, auch Kosaken genannt, anvertraut. Diese sorgten dafür, dass Russland eine schwingende Grenze zu seinen Nachbarn, den kasachischen Stammesverbänden, unterhielt. Diese „Schwingungen“ stellten ab der Mitte des 19. Jahrhunderts aber aus britischer Sicht die Grenze zu British Indien infrage und die Briten verlangten von Petersburg das Herstellen 
klarer Verhältnisse. ${ }^{1} 1867$ wurde das Generalgouvernement der Steppe gegründet und die russische Administration ging schrittweise dazu über, die Steppengebiete zu verwalten. ${ }^{2}$

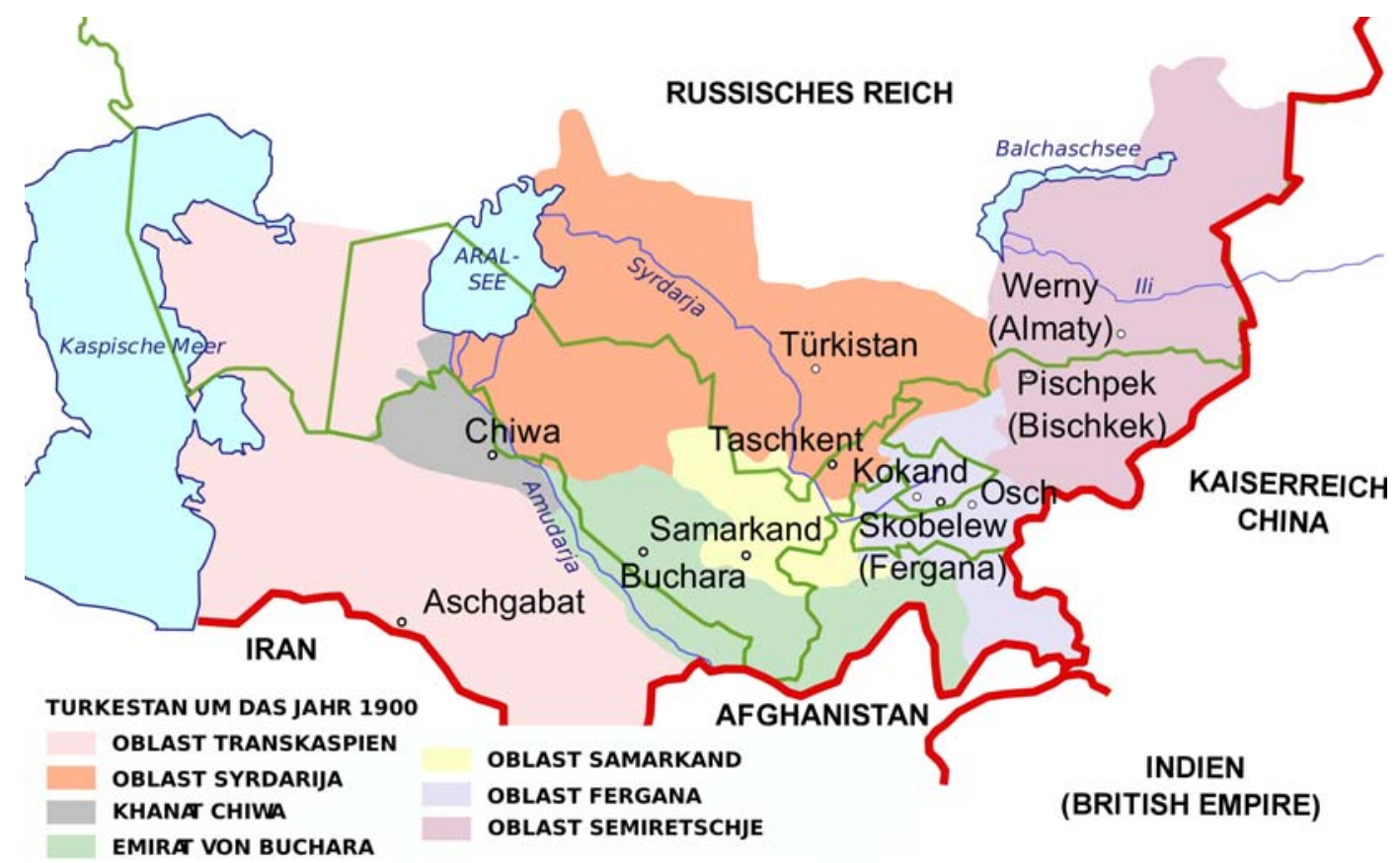

Abbildung 1: Die zentralasiatischen Bezirke des Russischen Imperiums. Schwerpunktregion dieses Artikels ist der östliche Bezirk Semirečie (violett).

In Russland war das 19. Jahrhundert darüber hinaus von Ereignissen bestimmt, welche die Modernisierung der Gesellschaft vorantrieben. Die Aufhebung der Leibeigenschaft 1861, die die Bauern schrittweise von der Schollengebundenheit befreite, war ein wichtiger Schritt zur Überwindung feudaler Strukturen. Die Freisetzung der leibeigenen Bauern 1861 führte zwar zu steigendem Wohlstand auf dem Lande, produzierte aber auch überall einen Mangel an bestellbarem Land. Die Übersiedlung nach Sibirien oder in die Steppengebiete wurde von vielen russischen Landlosen als probates Mittel gesehen und oft auf eigene Faust organisiert. Die Städte Omsk oder Orenburg, die ehemaligen Außenposten Russlands, wurden wichtige Anlaufpunkte der Kolonisierung neuer Gebiete in der kasachischen Steppe (Abb. 1). Die Interessen der Zentralmacht sollten in der Steppe ${ }^{3}$ durch

1 Der Begriff der schwingenden Grenze wurde von Theresa Elze eingeführt, siehe Elze, T.: Die Ambivalenz der Grenze. Handlungsorte an der deutsch-polnischen Grenze im Jahre 2006. Unv. Magisterarbeit am IfE der UL. Leipzig 2007, S. 71. Auf Zentralasien übertragen macht die schwingende Grenze darauf aufmerksam, dass Gebietsaneignungen in der Steppe und in Turkistan nicht immer auf Anordnung Petersburger Militärfunktionäre beruhten, sondern häufig durch den Eigensinn der dort stationierten Offiziere. Verwiesen sei auf die Annexion Taschkents am 5 Juni 1865 durch Oberst Chernjaev als prominentestes Beispiel.

2 Eine Abbildung, welche die Nationalallegorien „Bär“ und „Löwe“ illustriert, findet sich unter http://de.wikipedia.org/wiki/Russischer_B\%C3\%A4r_(Nationalallegorie).

3 Mit Steppe ist im gesamten Artikel das von der russischen Administration eingerichtete „Gouvernement der Steppe" gemeint. 
Gesetze abgesichert werden und die Grundlage für eine effiziente Kolonisation der Gebiete bilden. (Hildermeier 1989, 14-26)

Die Vorstellungen Petersburgs, Russland habe große, menschenleere Gebiete besetzt, wurden jedoch bald durch die Aufstände in den Steppen Lügen gestraft. Neusiedler hatten das Weideland nomadischer Viehzüchter besetzt und damit die Wirtschaftlichkeit der Nomaden in Frage gestellt (Škapskij 1907a, 134f). ${ }^{4}$ Landflüchtige Kasachen, meist durch Viehverlust zum Tagelohn getriebene Viehzüchter, suchten zudem Schutz und Lohnarbeit in den Steppenstädten Orenburg oder Omsk und verursachten hier eine Reihe sozialer Probleme. Die geplante Eisenbahnlinie, entlang derer Siedlungsstationen errichtet werden sollten und die das neue Generalgouvernement Turkestan mit den Zentralrussischen Gebieten verbinden sollte, wurde durch die Steppenaufstände in Frage gestellt.

In der russischen Kolonialpresse entbrannte daraufhin eine Debatte, wie diese Probleme in den Griff zu bekommen seien. Das Allheilmittel hierfür, so die einhellige Meinung, war die Sesshaftmachung der Viehzüchter. Um diese voranzutreiben und vor allem verarmten kasachischen Viehzüchtern eine Alternative zur Tagelöhnerei zu bieten, errichtete die russische Kolonialverwaltung Landwirtschaftsschulen in der Steppe. Sie sollten kasachische Viehzüchternomaden von ihrer Lebensweise abbringen und deren Weg in die Sesshaftigkeit durch Wissensvermittlung erleichtern. Dabei übersah die russische Kolonialmacht, dass die Kasachen durchaus über eine Feldwirtschaft verfügten. Diese wurde von verarmten Stammesmitgliedern in der Nähe der Winterquartiere betrieben.

Dieser Beitrag untersucht - um es vorwegzunehmen - die Geschichte eines gescheiterten Experiments, das Sesshaftmachung durch landwirtschaftliche Bildung erreichen wollte. Die Debatten, die diesen Versuch begleiteten, füllten 30 Jahre lang die Zeitungen in der kasachischen Steppe. Viele Justierungen, Verbesserungen und Anpassungen an die lokalen Gegebenheiten wurden vorgenommen, jedoch von den Kasachen konsequent missachtet.

Das Scheitern dieses Experiment zeigt, dass sich wirtschaftliche und damit kulturelle Eigenheiten an die Steppe knüpften, die in unvereinbarem Gegensatz zu den Gegebenheiten sesshafter Gesellschaften zu stehen schienen. Erst die gewaltsame Sesshaftmachung der Kasachen während der Kollektivierung in den 1930er Jahren führte letztlich zu dem von den russischen Verwaltern gewünschten Ergebnis. Eine Million Tote - etwa ein Drittel der nomadischen Bevölkerung - waren die Folge. (Ohayon 2006)

\section{Die Steppe}

Die Steppen Zentralasiens sind seit dem Trockenfallen des Inneren des Kontinents nach der letzten Eiszeit vor allem für eine nomadische Lebensweise geeignet. (Khazanov 1994, S. 94f.) Im Altertum lebten hier berühmte Reitervölker wie die Hunnen, die Saken oder die Mongolen. Nomadische Lebensweise bedeutet, die Steppe saisonal an verschiedenen Orten zu nutzen: im Sommer durch eine auf Schafen, Kamelen und Pferden beruhenden Weidewirtschaft, im Winter durch das Aufbrauchen der Überschüsse des Sommers. Dabei bedeutet ein großer Viehbestand Reichtum, Viehlosigkeit bzw. Viehverlust hingegen Armut. Bei den historischen Nomadenstämmen konnte ein begrenzter Anbau

4 Um z.B. den Verlust ihrer Winterweiden zu verhindern, blieben Viehzüchter manchmal im Sommer auf den kostbaren Winterweiden. Dazu ausführlich S. 68f. 
von Getreide im Sommer nachgewiesen werden, um für Nahrungsmittel im Winter, wenn die Milcherzeugung nachlässt, vorzusorgen.

Im meist regenreichen Frühling schlossen sich Haushalte zum Zug auf die Sommerweiden zusammen. Der Auszug in die Steppe garantierte den Haushalten ein reicheres Auskommen als die Sesshaftigkeit in den Winterquartieren. Nur wer seine Herde durch Krieg, Naturkatastrophen oder Misswirtschaft verlor, musste in den Winterquartieren verweilen. Sobald die Verarmten durch Stammeshilfe wieder zu Tieren gekommen waren, gingen sie wieder auf die Sommerweiden. Die Landwirtschaft der Stammesgruppen lastete somit auf den Schultern verarmter Stammesmitglieder und war Zeichen für einen Souveränitätsverlust im Stammesverband, sozialen Abstieg und Marginalisierung. (Botbaev 1890; Kurmanbaev 1894a)

Die Zusammengehörigkeit einzelner Haushalte wurde durch die Blutsbande geregelt, dem bestimmenden Merkmal tribaler Zusammenschlüsse. Es bildeten sich dadurch kleine Stammesgruppen, die sich hierarchisch um einen Oberhaupt (bij) gruppierten. Je nach Stärke der jeweiligen Stammesverbände vereinten sich mehr oder weniger Stammesgruppen um einen Führer, denen dadurch Landfrieden garantiert war, die sich aber auch an Verteidigungsaufgaben und -ausgaben zu beteiligen hatten. Rechtssprechung war interne Aufgabe der Haushalte, Gruppen bzw. Verbände, je nach Ausmaß des Konflikts. War eine Einigung auf Verbandsebene nicht erreichbar, zerfielen die Verbände durch Krieg. Die Stammesführerschaft war zwar erblich, wurde aber schnell aberkannt, wenn Charisma und Intellekt nicht für Führungsqualitäten taugten. Somit war Herrschaft in der Steppe sehr dynamischen Prozessen unterworfen und stützte sich auf ein komplexes System von Loyalität und Allianzen. (Lemercier-Quelquejay 1998, Tynyshpaev 1990)

\section{Die kasachischen Stammesführer und die Frage der Sesshaftigkeit}

Als das russische Zarenreich die Steppe eroberte, war es zunächst daran interessiert, die lokale Stammesaristokratie zu stützen. Das Toleranzgebot Katharina II. garantierte seit 1773 die Freiheit des religiösen Bekenntnisses. ${ }^{5}$ Für alle Religionsgemeinschaften wie den Islam, bei denen an das religiöse Bekenntnis auch an eine eigene Rechtsprechung gekoppelt war, bedeutete das zu einem gewissen Maß die Möglichkeit der Selbstverwaltung. Dieser regulative Rahmen wurde nur dann überschritten, wenn russischstämmige Personen in den Rechtsstreit verwickelt waren oder wenn der Streit russische Interessen berührte. (Wheeler 1964, 92f; Martin 2001, 134)

Bei den seit der Einführung des Steppenstatuts ${ }^{6}$ (1891) veranstalteten Wahlen von Gebietsvertretern und Richtern aus einheimischen Reihen konnten Stammesführer oft durch Loyalität, Stimmenkauf und diverse politische Tricks zu einflussreichen Personen werden. In einem Artikel beklagt ein kasachischer Autor, dass die früher gelobte Weisheit der Stammesführer durch persönlichen Reichtum mehr und mehr untergraben werde. Nur

5 Das regelte das Toleranzedikt vom 17. Juni 1773. Davon ausgenommen war allerdings die große Zahl von Juden, die seit der Ersten Teilung Polens ihre Untertanen waren. Siehe: Innenpolitik von Katharina II in der wikipedia (http://de.wikipedia.org/wiki/Katharina_II._(Russland)\#Innenpolitik 16.08.2009).

6 Das Steppenstatut ist 1890 das erste für das Generalgouvernement der Steppe verabschiedete Gesetzeswerk, das die administrativen Rechte der Kolonialregierung in der Steppe regelte. 
Reichtum könne einen Posten in der Verwaltung garantieren. Es gäbe sogar Lieder, die besingen: „Du kannst einen Verstand haben wie Platon, doch an den Gebietsposten kommst du ohne Geld nicht.“ (Kurmanbaev, 1894b)

Neben Eingriffen in die nomadische Selbstverwaltung wollte die russische Kolonialregierung aber auch Einfluss auf die sozialen Strukturen der Steppe nehmen. Als der Semirečie-Gouverneur Kolpakovskij beschloss, die zur Bewachung der Grenze wichtige Kosakenarmee zu vergrößern, eröffnete er den Kasachen die Möglichkeit, in die Kosakenregimenter einzutreten. Die Sesshaftmachung einzelner Haushalte hieß jedoch automatisch Gebiets- und damit Machtverlust der Kasachischen Stämme. (Čirkin 1907, 73) Durch die staatliche Vergabe von Ackerland an Nomaden hoffte man, diese aus dem Einflussbereich der kasachischen Stammesführer herauszuholen und sich eine Masse loyaler Bauern zu schaffen. Die Beamten vor Ort beteuerten, dass von einzelnen Armen mehrfach der Wunsch geäußert worden sei, Land zu erhalten, um sich von den Stammesoberhäuptern lossagen zu können. (Cabel' 1907, 79-100) Damit schufen sie ein Problem: Je mehr Nomaden sich im administrativen Gebiet, in dem sie gewählt werden wollten, sesshaft machten, desto mehr Stimmen verloren sie, da nun Loyalitäten wechselten. Durch das Machtinstrument der traditionellen Gerichte und der Selbstverwaltung konnten sie immer wieder durchsetzen, dass sich Leute aus den Listen, die Freiwillige für die Ansiedlung mobilisieren sollten, wieder austrugen. (Čirkin 1907, 76f)

\section{Das russisch administrative Interesse an der Sesshaftigkeit}

Eines der simpelsten Argumente der Kolonialadministration, die Sesshaftigkeit zu propagieren, war die Erleichterung der Arbeit auf der administrativen Ebene, da man eine sesshafte Gemeinde eindeutig in ihren Grenzen abstecken konnte. Die ständige Bewegung, die die nomadische Lebensweise mit sich brachte, bedeutete für den Fiskus steuerliche Unsicherheit. Nomaden erschwerten es der Administration, ihr wirkliches Steueraufkommen zu schätzen und einzutreiben. Die steuerliche Last für sesshafte Bauern hingegen konnte man eindeutig an deren Ertrag messen. (Škapskij 1907b, 46) Sesshaftigkeit kam dem Staat finanziell zugute, da sich die Steuereinnahmen in Bezirken mit Tendenz zur Sesshaftmachung erhöhten. (Čirkin 1907, 74)

Die sesshaft Gewordenen schufen außerdem Platz für die nachrückenden Siedler, da ihr Platzaufwand scheinbar viel geringer war. Die koloniale Administration konstatierte: Für hundert Pud Weizen brauche man einen Hektar Land, der fünf Menschen problemlos ernähren könne. Für vier Stück Vieh brauche man hingegen 25-30 Hektar Land und die könnten nur einen Menschen ernähren. (Čirkin 1907, 65) Die Sesshaftigkeit galt auch als Lösung für die Probleme des Land- und Viehdiebstahls. Die ständigen Kämpfe zwischen Stammesgruppen um die besten Weideplätze, die damit verbundene ,administrative Instabilität" in den kasachischen Gebieten und die immer wieder neuen Kräfteverhältnisse in der administrativen Ordnung sollten hierdurch umgangen werden. (Rumjancev 1912, 158f; Kurmanbaev, A. 1894a)

Es gab Kasachen, die sich tatsächlich auf russischem Land sesshaft machten und die Loyalitäten wechselten. Ein Beispiel für die Ansiedlung kasachischer Nomaden im Süden (Bischkek) zeigt dies deutlich: Hier wurden jedem freiwilligen kasachischen Siedler elf Hektar Land gegeben. Die Bewirtschaftung sah nur eine Produktion für die örtlichen staatlichen Abnahmestellen vor, der Staat war also einziger Abnehmer der Ernte. Die Freiwilligen bekamen Maschinen und Saatgut vom Staat gestellt und mussten diese Schuld innerhalb von zehn Jahren abbezahlen. Sie wurden aus ihren traditionellen Ge- 
meinschaften ausgeschlossen, die sich nun nicht mehr für sie verantwortlich fühlten und bildeten eine eigene Gemeinde, die sich auf das russische administrative System und die russische Gerichtsbarkeit berief. (Čirkin 1907, 74)

\section{Pläne zum Ausbau des Gebietes mit Hilfe der Eisenbahn}

Wichtige Impulse zur Sesshaftmachung gingen neben den administrativen Maßnahmen vom Projekt des Eisenbahnstreckenausbaus in Russland aus. Die ersten großen Siedlungsbewegungen in den 1860er Jahren waren vor allem entlang der neuen Eisenbahntrassen in Sibirien zu finden. Die Eisenbahn wurde schnell zum wichtigsten Transportmittel für Rohstoffe aus den kolonisierten Gebieten und für Menschen, Lebensmittel sowie Haushalts- und Wirtschaftswaren nach Sibirien. (Stepnoj 1909, 244) Die Apologeten des Eisenbahnbaus brachten ihre Intensionen deutlich zum Ausdruck: Die Kolonisierung könne nicht auf Landverteilung oder auf den Transport zum Neuland reduziert werden. Mit dem Bau der Eisenbahn sei der Staat ebenso zu einer Reihe von Kulturarbeiten genötigt: Wege-, Brunnen-, Krankenhäuser- und Schulbau. Mit der Änderung der Politik seitens der Regierung kämen die Siedler in großen Wellen immer weiter in den Süden, in die Tiefen der Kasachischen Steppe. Da die meisten Siedler aber schon außerhalb des Einflussgebietes der Eisenbahn geraten seien, bedürfe es nun neuer Linien in die Steppe hinein. (Stepnoj 1909, 245 249) Den Befürwortern des Eisenbahnbaus war es weniger wichtig, welche Plätze die Nomaden belegten und welche Wirtschaft sie dort führen konnten. Was sie interessierte, war die Bereitstellung von zusammenhängenden Ländereien, damit sich entlang der Trasse Bauern ansiedeln konnten, die eine Rentabilität der Eisenbahn garantierten. Die Argumentation mit Zahlen war ihnen die wichtigste. ${ }^{7}$ Die Eisenbahnpioniere selbst benutzten noch radikalere Argumente. Sie schlugen vor, in Gebieten, die Möglichkeiten zum Ackerbau böten, im Zuge einer Landreform den kasachischen Nomaden Land auf der Grundlage von Ackerbaunormen zuzuteilen. So konnten diese zur Sesshaftigkeit gezwungen werden. Bei Unwilligen nähme man in Kauf, dass sie Richtung Süden auswandern. (Uspenskij 1907, 41f) Die Schiene prophezeie der Steppe blühende Zeiten, da die Eisenbahn Möglichkeiten zum Aufbau Industrieansiedlungen schaffe, wie Leder- und Wollbetriebe, Weinanbau, Mühlen und Bergbau. Außerdem bringe die Eisenbahn eine Reihe von Investoren mit sich, die auch aufgrund der in Russland vorherrschenden Industrie (Landmaschinen, Düngemittel, Material zur Etablierung neuer Industriestandorte usw.) Sesshafte als Kunden brauchten. (Čirkin 1910)

\section{Die Siedler}

Als die Politik Alexander II. den leibeigenen Bauern 1861 mehr Freiheiten gab, musste der Russische Staat in der Siedlungspolitik unverzüglich handeln. Hunderttausende

7 In dem Bericht Čirkins, einem wichtigen und einflussreichen Ökonomen, stellt er für das Komitee der Sibirischen Eisenbahn 1910 fest: Die Normen der Landnutzung für die Kasachen sei viel zu hoch angesetzt worden in den neunziger Jahren. Man sei von falschen Wirtschaftsgrößen ausgegangen: nicht 17-19 Personen pro „Haushalt“ sondern 24. Man machte bei den Normen dazu noch einen Aufschlag von 25\% als Puffer. Aus lauter „Rücksichtnahme“ besetzte man die „freiwerdenden“ Plätze nur zu 2/3. Die Einheiten der Besiedlung wurden auf der Ebene des Volostes eingeführt und sind so zu sehr zerstückelt und wenig rentabel für eine Eisenbahn. (vgl. Čirkin 1910, 34-43) 
Siedler gingen jedes Jahr über den Ural und siedelten sich ohne jede staatliche Regulierung auf Landflächen an, welche sie vorteilhaft für den Ackerbau hielten. (Xvorostankij 1907, 61) Im Zuge der schrittweisen Eroberung der Steppe nahmen sich die Kosaken das Land, welches sie benötigten, um ihren Eigenbedarf abzudecken. Land war auch der gängige Sold unter den Truppen, die die Grenzen und das eroberte Gebiet des russischen Imperiums sichern sollten. So bekamen im Siebenstromland (Semirečie) von 1847 bis 190530 Siedlungen der Kosaken Land für den Ackerbau. 1905 waren etwa 32.000 Kosaken in diesem Bezirk. Von 1868 bis 1882 kamen die ersten russischen Siedler und konnten hier in Semirečie 28 Siedlungen gründen. Von 1882 bis 1905 kamen noch mal acht Siedlungen hinzu. Die große Welle der Besiedlung setzte dann 1906 ein. (Demko 1969, 44) Die Siedler kamen selten aus einem abenteuerlichen Pioniergeist in die Region, sondern oft aus nackter Not. Landarmut und Hungersnöte spielten hier wohl die größte Rolle. So war der Strom der Siedler zur Zeit der großen Hungersnöte, der Februarrevolution und dem verlorenen Russisch-Japanischen Krieg 1905/6 am stärksten. (Demko 1969, 78)

Die Siedler nahmen sich das Land entweder gewaltsam von den Einheimischen oder pachteten es von Nomaden für einige Jahre. ${ }^{8}$ Durch die willkürlichen Landnahmen sprach sich herum, dass es im Südosten Russlands ein Leichtes sei, an Land heranzukommen. (Čirkin 1907, 60) Die oft praktizierte gewaltsame Landaneignung durch die Siedler war nicht zuletzt deshalb möglich, weil Kasachen sich vor Zusammenstößen mit Russen eher hüteten. Sie hatten im Falle eines Streites auf rechtlicher Ebene die schlechteren Karten, da hier nicht mehr ihr Gewohnheitsrecht, sondern das russische Recht galt, in dem sie keine Spezialisten waren und von daher leichter betrogen werden konnten. Die örtliche Administration und die Siedlergruppen gingen mit den Rechten der Kasachen rücksichtslos um. Bis in die Mitte des 19. Jahrhundert wurden bei der Landvergabe für Kosaken und russische Bauern die Interessen der Nomaden kaum wahrgenommen. Oft wurden sogar Ländereien veräußert, die schon von Nomaden bewässert wurden. (Škapskij 1907b, 21-27) $)^{9}$ Die Situation verschärfte sich mit den Jahren und den immer stärker werdenden Siedlerströmen. Im Jahre 1906 wurde von der aktuellen politischen Lage in der Steppe folgendes berichtet: Die Kasachen seien auf ihren Winterweiden geblieben, um eine Veräußerung dieser an die Siedler zu verhindern. Als Siedler in das Gebiet kamen und keine freien Plätze mehr fanden, hätten sie sich das Land gewaltsam angeeignet. Es sei zum Blutvergießen auf beiden Seiten gekommen. (Škapskij 1907a, 134)

8 Siedler pachteten bei Kasachen Land oder siedelten sich schon bei vorhandenen russischen Siedlungen an, ohne Land zu besitzen. Viele von ihnen hatten kein Land am Hof, sondern bestellten Felder, die bis zu 15-20 Werst von den Wohnorten entfernt lagen. Die landlosen Bauern warteten auf die Landvergabe oft einige Jahre, sie fanden also dieselbe Situation vor, vor der sie aus den Zentralrussischen Ländereien geflohen waren. Dabei müssen aber die Regionen voneinander unterscheiden werden: Im Nord-Osten Siedelnde mieteten sich jährlich ihre Felder bei Kasachen oder Kosaken an. Sie lebten vor allem in Wohnungen, nur etwa $25 \%$ besaßen ihre eigenen Höfe. Viele von ihnen wanderten in die Gegend von Bischkek $\mathrm{ab}$, wo die Situation völlig anders aussah. Hier wohnten nur 25\% in Wohnungen und 37\% hatten ihre eigenen Höfe, etwa 15\% siedelten sich in der Stadt an. (Škapskij 1907a, 112-137)

9 Die Landnahme der Siedler geschah oft mit Begründungen wie: „Die sind ja nicht getauft.“ oder „Sie können ruhig in der Hitze leben.“ oder später „Sie kämpften ja nicht gegen die Japaner.“ (Čirkin 1907, 61) 


\section{Landvergabe - die Rolle des Staates in der Siedlungspolitik}

Im Steppenstatut von 1890 wurden für die Belange des Landeigentums und der Besiedlung u.a. zwei wichtige Grundsätze festgelegt:

„1. §119 Das Land, welches von den Nomaden belegt wird, darin eingeschlossen der Wald [...], wird zum staatlichen Eigentum gezählt.

2. $\$ 120$ Das Land, welches von den Nomaden belegt wird, bleibt in unbefristeter gemeinschaftlicher Nutzung der Nomaden, auf den Grundlagen der Regeln und Bräuche ihrer Rechtsprechung.

Bemerkung: 1. Das Land, welches für die Nomaden als überschüssig betrachtet werden kann, geht in die Aufsicht des Ministeriums für Staatseigentum über. [...]" (Masevič $1960,395)$

Für die Verwaltung des ,überschüssigen“ Landes der Nomaden wurde das „Ministerium für die Verwaltung des Staatseigentums“ beauftragt. Auch für die Lösung der Frage der Landverteilung wurden Institutionen gegründet, die die Landvergabe an Siedler, aber auch an Kasachen, organisierten.

Die Frage staatlich angeordneter Veräußerung von Nomadenland im großen Stil berührte empfindlich die Frage kasachischer Wirtschaftsformen. Denn mit der Frage der Landflächennormen pro Produktions- und Lebensgemeinschaft verband sich auch die Frage, welchen Platz man der extensiven Viehzucht einräumen wollte. So entsandte man 1894 eine Expedition in die Steppe, die Grundlagen und Formen der kasachischen Landnutzung, die ökonomischen Faktoren einer durchschnittlichen kasachischen Wirtschaft und die natürlichen Gegebenheiten verschiedener Kreise erforschen sollte. Sie sollte Normen einer Landnutzung für eine mittlere Produktions- und Lebenseinheit (sprich: Familie) aufstellen. (Xvorostankij 1907, 62f, Demko 1969, 57) Die Fragen, mit denen sich die Expedition beschäftigte, fördern deutlich zu Tage, wie wenig einige Jahrzehnte nach der Eroberung der Steppe den russischen Kolonialbeamten die Wirtschaft der kasachischen Viehnomaden vertraut war. Auch waren die natürlichen Bedingungen, die eine landwirtschaftliche Nutzung erst möglich machten, weitgehend unbekannt.

\section{Die Sesshaftigkeitsdebatte: Durch Bildung zum Erfolg?}

Die politischen und wirtschaftlichen Kämpfe um die Besiedlung der Steppe bilden den Hintergrund einer Sesshaftigkeitsdebatte ab den 1890er Jahren. Die russische Kolonialelite musste die Sesshaftmachung von Nomaden zunächst mit der Macht der Überzeugung erkämpfen, da die russische Lokalmacht in der Steppe noch auf zu wackeligen Füßen stand, um bei ernsthaften Auseinandersetzungen mit den kasachischen Stammesverbänden die Oberhand zu behalten. Zu Hilfe kamen ihr dabei die Aufklärer aus den Reihen der Kasachen, die Sympathien für die Errungenschaften der russischen Zivilisation hegten und versuchten, ihre Stammesgenossen vom Wert der Sesshaftigkeit zu überzeugen. Im Folgenden sei ein Beispiel für eine solche Position zitiert:

„Alle jetzt aufgeklärten Völker waren früher einmal so wild wie wir, die Kasachen, es heute sind. Sie sind umhergezogen, hatten keinen bestimmten Platz, an dem sie ständig wohnten und bestahlen sich gegenseitig. Es gab bei ihnen keine Gesetze, keine Rechte. Doch jetzt sind sie weit in Bildung und Aufklärung fortgeschritten. [...] Unter solchen [umherziehenden] Völkern befinden auch wir uns, wir Kasachen, und uns ist es sehr pein- 
lich, dass wir bis jetzt immer noch so wild und ungebildet sind, obwohl wir uns schon an die 100 Jahre unter russischer Herrschaft befinden, obwohl die russische Regierung sich unser annimmt und sich um unsere Bildung kümmert und uns Zutritt zu verschiedenen schulischen Einrichtungen ermöglicht. Warum bloß rennen wir weg vor solcher Bildung?

Für uns ist die Bildung und Aufklärung etwas unbedingt Nötiges und Wichtiges. Schaut Euch doch einmal um, wer jagt uns nicht alles Angst ein und belügt uns? Die Kosaken und die Bauern blicken auf Euch herunter, die Sarten ${ }^{10}$ und die Tataren belügen Euch und benutzen euren natürlichen Reichtum. Wer von Euch weiß nicht, dass die Tataren, welche vor ihrer Wehrpflicht flüchten, sich bei Euch ansiedeln, sich in Eurer Wirtschaft einnisten und endlich sich als Verkäufer betätigen, passiert das etwa selten? Irgendwelche Sarten, Dahergelaufene aus Kaschgar oder Taschkent, welche dort noch nicht mal gemeine Arbeit ${ }^{11}$ finden könnten, betätigen sich hier als Kaufleute und kaufen unsere Schafe zu sehr niedrigen Preisen. Nun, vielleicht kann man sagen, wenn wir morgens und abends unsere eigene ,Haut ${ }^{12}$ essen, auf den Winterweiden uns mit Stutenmilch (kumys) vergnügen, wozu brauchen wir dann noch Bildung? Doch bitte, so ist es ja nur zum Teil. Weil wir Kasachen uns nicht immer über die Situation, in der wir uns befinden, freuen können, ergehen wir uns in Neid über den Reichtum der Fremdländischen. Wir sind nicht immer zufrieden mit unserer ,Haut' und unserem Kumys, um so mehr sie sich nicht bei jedem in ausreichender Menge befinden. Und dann sehen wir, dass die Russen, die Tataren und die Sarten jeden Tag Tee trinken und Fleisch essen. [...] Uns reichen die Mittel dafür nicht aus und wir schauen neidisch auf die Glücklichen und beklagen: ,Nun, die befinden sich in guter Verfassung, doch wir nicht ${ }^{\star}$. Schaut doch einmal auf die Tataren, auf die Sarten, die Kosaken, wie ordentlich und sauber sie angezogen sind und wie sauber und ordentlich bei ihnen alles ist! Bei uns gibt es nichts Sauberes und Ordentliches. Bettwäsche benutzen wir ein Jahr, d.h. eigentlich solange, bis sie kaputt ist. Unsere Kleider sind staubbedeckt und fettig. Die Winter verbringen wir in Jurten, in denen wir vor Kälte und Hunger einfach wegsterben. Es zeigt sich, dass wir nicht genug haben, nicht einmal Heizmaterial oder Kleidung. Das passiert in einem normalen Winter. Aber in einem Winter mit überfrierendem Eis $(d z ̌ u t)$ ? Oh helfe uns Gott, ist es noch um vieles schlimmer. [...] Wir sterben weg wie die Tiere und keiner hilft uns, aber wenn uns jemand hilft dann sind das die Wunderheiler (bakši), die selber keine Ahnung haben. Doch wir glauben diesen Heilern, aber warum glauben wir keinen gelehrten Doktoren? ${ }^{13}$ [Es folgen noch einige Bemerkungen zu (tatarischen) Schwindlern, u.a. einem Advokaten, der selber Analphabet war. (Anm. d. A.)]

Doch wären wir ein wenig gebildet oder erfahrener, dann würden uns solche ,Advokaten' nicht mehr betrügen; dann wären wir immer satt, hätten immer anzuziehen, wären sauber, hätten den Handel in unseren Händen; Schafe, Pferde, Großhornvieh würden wir selber auf den Markt treiben und nicht die Tataren oder die Sarten. Wir hätten eigene Läden usw. Also gebt Eure Kinder in die schulischen Einrichtungen dieser Steppe und Ihr werdet sehen, dass aus ihnen Kinder des Gerichts, Entdecker und Doktoren werden.“ (Appasov 1895)

10 Kolonialbezeichnung für alle Sesshaften, Bauern wie Städter, aus dem Generalgouvernement Turkistan.

11 Meint hier wohl „Zeitarbeit“ als Tagelöhner.

12 Meint hier wohl autarkes Leben, ohne Abhängigkeit von Märkten bzw. externer Versorgungslage.

13 „Traditionelle Medizinmänner und gelehrte Doktoren“ beziehen sich hier offenbar auf die Gegenüberstellung von traditionellen und europäischen Gelehrten. 
Bei den kasachischen Aufklärern lassen sich einige Ansatzpunkte finden, die in den Debatten von russischen Bildungspolitikern und Kolonialbeamten so nicht vorhanden waren. Die Suche nach einem kasachischen Selbstbewusstsein war eines ihrer zentralen Anliegen. Um als Volk einen höheren Zivilisationsgrad zu erlangen, um sich nicht schämen zu müssen vor den anderen Völkern, sollte ein jeder mehr Bildung erlangen. Obwohl die Minderwertigkeit des eigenen Volkes im Vordergrund stand, schwang ständig ein Unterton mit, der ein gewisses „Selbstbewusstsein“ zu postulieren versuchte. Der Autor, Sultan Appasov, war kein blinder Lobessänger der russischen Bildung, sondern formulierte vor dem Hintergrund der Debatte um die vermeintlich verpassten wirtschaftlichen Chancen, die eigene Trägheit und relative Unwissenheit auch folgenden Gedanken: Wären die Defizite von europäischer Bildung schon ausgeglichen, um wie viel besser würden die Kasachen dann dastehen? Um wie viel überlegener, hoffte er, wären sie den „Dahergelaufenen“ und „Scharlatanen“? (Appasov 1895)

Eine andere Gruppe der Debattierenden waren aufgeklärte, sozialrevolutionär denkende Russen, die vor allem die Armen (džatak) der Steppe ansprachen, weil sie hier die dankbarsten Empfänger für ihre Kulturmission sahen. Eine ganze Zeitungsrubrik war ihnen gewidmet - „Zur Verbesserung des Lebens der landlosen Viehzüchter“ (O ulučenia byta džatakov) - in der es um die Propagierung von Bildung und Ackerbau ging. In ihren Statements überwog eine sozialkritische Herangehensweise. Viele ihrer Artikel machen klar, dass sie Menschen formen wollten. Aus ihrer Perspektive bildeten die Armen die leicht formbare Masse. Ihre fast missionarischen Schriften lesen sich so:

„Einem, dem noch nicht das Leben der Kasachen der Steppe bekannt ist, muss das Leben von ihnen nicht nur eigenartig, sondern sogar wild erscheinen. Die Zivilisation hat sie so wenig berührt, jene die Freiheit gewöhnten Bewohner der Steppe, dass man denken kann: ,Nein, die wirst du niemals ändern!' Sogar die Kasachen, die in russischen Bildungseinrichtungen waren, bleiben in der Seele die gleichen, die sie schon vor Schuleintritt waren. Sie verbringen mit großem Vergnügen lieber ihre Zeit in ihren Jurten, als in schönen Zimmern. Das Nomadenleben ist ihnen lieber, als das der Stadt und der Siedlung, sie schätzen Letzteres [die Städte und Siedlungen der Sesshaften (Anm. d. A.)] auch nicht und die erste Gelegenheit, die sich ihnen bietet, nehmen sie wahr, um zum alten Leben zurückzukehren. Der Kasache in der Stadt, ganz egal ob Vogel im Käfig ${ }^{14}$ oder nicht, fühlt sich an Armen und Beinen gebunden durch das, was wir eine Wohltat nennen. Doch es gibt Ausnahmen, aber die sind nicht sehr zahlreich." [Es folgen Fallbeispiele sesshaft gewordener Kasachen (Anm. d. A.)] (DVG/KSG 43, 1895)

Es waren aber auch Stimmen zu hören, die ganz pragmatisch an die Sache herangingen: Wer weniger Land braucht, hat mehr Berechtigung auf dessen Nutzung als ein anderer.

„Die alljährliche Übersiedlung von Siedlern in die Steppenbezirke aus den zentralrussischen Gouvernements macht die Bereitstellung von neuen Plätzen zur Besiedlung und dazu die Abführung von passenden Landstücken aus den Ländereien, die Staatseigentum sind, die aber zur jetzigen Zeit in kasachischer Nutzung stehen, erforderlich. Für die Bil-

14 Meint hier wahrscheinlich: $\mathrm{Ob}$ nun eingesperrt oder nicht. 
dung neuer Siedlungen braucht es natürlich solche Plätze, die eine gute Qualität für eine bäuerliche Wirtschaft haben: die Nähe zu ausreichend Wasser, Wäldern, fruchtbaren Böden, eine ausreichende Anzahl von Weideland zur Heugewinnung, einfache Erreichbarkeit usw. [...]

Da die Anzahl der Bauern in den zentralrussischen Gebieten stetig wächst und es dort kein freies Land mehr gibt, wird es notwendig sein, in den Teilen des Landes Ackerland zu verteilen, wo es sich als frei erweist. Daher wird es früher oder später im Interesse des Landes sein, die Teile, die jetzt noch den Kasachen gehören, in den Besitz von Siedlern zu übergeben. Natürlich stößt es auf den Unmut von Leuten der Steppe, wenn aus ihrem Besitz Land genommen wird. Jeder ist traurig, wenn er Land hergeben muss, auf dem er, vielleicht jahrelang, Viehzucht betreiben konnte, aber was soll man tun? - Es darf doch nicht nur im Interesse des gesamtgesellschaftlichen Allgemeinwohls, sondern auch im Interesse der einfachen Gerechtigkeit sein, dass in einem Teil des Landes die Landbewohner nichterwähnenswerte Fetzen Land besitzen, der die Familie nicht ernähren kann, in anderen Teilen jedoch einzelne Familien zu hunderten Hektar Land besitzen.

[...] So gibt es für die Viehzucht nur zwei Möglichkeiten, entweder, die Fruchtbarkeit oder Erträge aus der Erde zu erhöhen, so dass weniger Land mehr Ertrag bringt, oder die Qualität des Viehs erhöhen, so dass weniger Vieh mehr einbringt. Welchen Weg der Kasache auch wohl wählen mag, ihm bleibt wohl nicht der Weg in die Landwirtschaft erspart, denn anders ist es ja nicht möglich, die Erträge der Erde oder die Qualität des Viehs zu erhöhen. Um den Übergang zur neuen Ordnung zu erleichtern, sollte man früh genug damit anfangen, sich dafür zu interessieren, da ansonsten immer weniger Platz zur Viehzucht zur Verfügung stehen wird, denn die beste Erde wird dem zustehen, der am besten auf ihr arbeiten kann. Beschwerden über die Konfiszierung von Erde aus ihrem Besitz können gar kein Mitleid erwecken, denn wem würde man schon Land zusprechen, wenn zwei sich darum bewerben und einer zehnmal weniger Land als der andere fordert und besser darauf leben kann als der andere - wem würde man es wohl bevorzugt geben?“ (DVG/KSG 10, 1894)

\section{„Kasachische“ vs. „russische“ Bildung}

$\mathrm{Zu}$ Beginn der russischen Eroberung und der schrittweisen Etablierung russischer Verwaltungsstrukturen in der Steppe schrieb die zuständige Kommission für die Regierung der Steppe, Kasachen hätten einen relativ großen Wissensdurst und seien, wenn sie das Geld dazu haben, auch in der Lage, ihren Kindern Bildung zu vermitteln. Dies geschehe über Hauslehrer, die über ein gut funktionierendes Ausbildungssystem verfügten. Neben der traditionellen islamischen Grundausbildung durch religiöse Würdenträger der Steppe gab es aber auch Akteure, die sich moderner Bildung und Aufklärung verschrieben hatten. Dieses Ausbildungssystem, wurde „tatarische Volksbildung“ (Girs et al 1960) genannt.

Die russischen Bildungspolitiker hatten dieser Form der Bildung gegenüber Vorbehalte, die sich auch in den Artikeln der Steppenzeitung niederschlugen. Dort wurde nicht nur von der angeblichen Unehrlichkeit der Tataren und deren betrügerischen Machenschaften berichtet, sondern auch von religiösem Fanatismus. Es wurde versucht, dieses „Problem“ 
durch bestimmte Kontrollmechanismen, wie z. B. die Einführung einer Ausweispflicht für Lehrer, in den Griff zu bekommen. ${ }^{15}$

„Bald ist es zu spät, wenn die Tataren mit ihren Schulen die gesamte Bevölkerung fanatisiert haben und in die Massen solche Vorurteile eingepflanzt haben, welche auf dem Weg zur wirklichen Aufklärung stören. Jetzt muss man die Verbreitung der speziellen Bildung, z.B. der landwirtschaftlichen, zunächst aussetzen, wenn das Volk vor allem erst mal eine allgemeine Bildung und deren Verbreitung benötigt. Macht den Kasachen erst mal zu einem guten Menschen, danach kann man aus ihm einen Bauern oder einen Handwerker machen.“(DVG/KSG 42, 1895)

Natürlich gab es unter den Kasachen Vorbehalte gegen die Bildungsversuche ihrer Kolonisatoren. Sie waren zum einen auf der Konfessionalität begründet, da durch die fehlende Unterweisung in islamischen Belangen die Schulen inakzeptabel für einen groBen Teil der Bevölkerung waren. Eine traditionelle Bildung sah vor, dass Schüler auch die islamischen Riten und Gebete erlernten und weitergeben konnten. ${ }^{16}$ Auch traute man den russischen Bildungspolitikern nicht, da Zwangstaufen in Waisenhäusern sowie Missionierung und Russifizierung in den 1870er Jahren sattsam bekannt waren. Wer gab die Garantien, dass Kindern nichts derartiges passierte, während sie in die russischen Schulen gingen? (B-OV 1895)

Warum schickten einige Kasachen ihre Kinder trotzdem in eine russische Schule? Das Hauptmotiv, den eigenen Kindern russische Bildung angedeihen zu lassen, lag in der Möglichkeit, damit eigene Interessen gegenüber russischen Institutionen besser durchsetzen zu können. Die Kinder der Viehnomaden sollten Zugang zu den Kanzleien zu finden oder als Wundärzte oder Viehdoktoren in der Steppe zu Ansehen kommen. Das bedeutete Spezialisierung aber auch Wirtschaftswechsel. Dieser war jedoch nur einer reichen Elite vorbehalten und deren Zahl war natürlich sehr klein.

Russische Bildungspolitiker wiederum wollten sich Untertanen „erziehen“, die erstens Russisch sprechen und zweitens die Rechte und Pflichten eines russischen Staatsbürgers kennen und annehmen. (DVG/KSG 33, 1891) Die russischen Vorstellungen, wie den „wilden Nomaden“ etwas beizubringen sei, unterschieden sich von den Erwartungen, die an die sesshafte Bevölkerung gestellt wurden. Man glaubte, dass die Kasachen weniger bereit und aufnahmefähig für russische Bildung seien, als die sesshaften Bauern Turkistans (sart) und wollte für sie in den Landkreisen Schulen des „niederen Typs“ einrichten. (Bart'old 1963, 298) Diese sollten eine praktische Ausrichtung haben, stärker das Handwerk, die Landwirtschaft, den Gartenbau oder die Forstwirtschaft fördern und nicht so viele Klassenstufen haben. Die Idee zum Aufbau von Landwirtschaftsschule war entstanden.

In den siebziger Jahren wurden in den verschiedenen Kreisstädten des Steppengebietes Internate errichtet, die speziell für Nomadenkinder geschaffen wurden. Doch nur wenige

15 Glosse über die gierigen Tataren, die als Hauslehrer Nomaden arm machen (Džapakov, K 1895), Erörterung des Zurückdrängens des Einflusses der Tataren (DVG/KSG 23, 1895), Frage der Ausweise (S. M. Č. 1890)

16 In der Zeitung „Dala Viloyati Gazeti“ wurde gegen diese religiösen Vorbehalte agitiert und es wurden Beispiele aufgezählt, wo ehrbare Muslime westliche oder russische Schulbildung aufgenommen hätten, ohne Gewissensbisse oder Vorbehalte. (Imankulov 1894) 
von denen, die die Schule besuchen wollten, konnten tatsächlich aufgenommen werden, da die Internatsunterbringung nicht kostenlos war. Die Schulabgänger dieser Kreisschulen konnten dann weiter nach Omsk gehen, um hier auf einer Wundarztschule oder in anderen schulischen Einrichtungen weitere Bildung zu erlangen. (Bekodžin 1895) Bis in die 1880er Jahre hinein existierten Internate für kasachische Kinder noch, doch dann wurden sie abgeschafft. Was konnten die Kasachen jetzt tun, um ihre Kinder „russisch“ bilden zu lassen? Übrig blieb nur noch die Unterbringung in einer städtischen Wohnung, welche viel Geld kostete. Auch musste vorher die russische Sprache erlernt werden, da in den städtischen Schulen auf Russisch unterrichtet wurde. Reiche Kasachen konnten sich Abhilfe schaffen, indem sie ihre Kinder zu Kosaken in den „Unterricht“ gaben, was allerdings auch Kosten verursachte. Obwohl reiche Kasachen über viel Vieh verfügten, gab es wenig hartes Geld in ihren Haushalten, da der innerkasachische Handel vom naturalen Tauschhandel bestimmt war. (Omskij Kirgiz 1895)

Die Frage der Lehrinhalte wurde oft diskutiert und es prägten sich mehrere Richtungen aus. Es gab Vertreter der allgemeinbildenden Schulen, es gab aber auch Anhänger der landwirtschaftlichen Schulen. Dass sich die Vertreter der letzten Richtung Ende des 19. Jahrhunderts durchsetzen konnten, legt folgendes Zitat nahe:

„Lieber früher als später anfangen, zur Sesshaftigkeit überzugehen, da ansonsten die Geldmittel so niedrig sind, dass man die finanziellen Möglichkeiten für diesen Schritt nicht hat. [...] Daraus ergibt sich das klare Resultat, dass eine solche Schule wie die landwirtschaftliche Schule nach den gegebenen Bedingungen die einzig sinnvolle ist, ohne Zweifel, denn wenn die gesamte kasachische Bevölkerung den Getreideanbau nicht erlernt hat, wird sie in der Zukunft genötigt sein, von der Viehzucht zum Ackerbau überzugehen, ungebeten, als präventive Maßnahme, um ihrer Existenz und den Ausbau ihrer wirtschaftlichen Bedingungen willen. Bis die kasachische Bevölkerung diesen Schritt nicht vollständig vollzogen hat, ist jede andere Art der Bildung für die kasachische Bevölkerung unnötig. Wofür soll der Kasache die Abstraktheit, das reine theoretische Wissen, welches er auf einer ganz normalen Schule erhält, anwenden zwischen seinen Stammesgenossen? Zu nichts anderem als Eingaben an die Leitung zu schreiben! Die theoretische Schule entfernt den Kasachen nur von seinen Mitmenschen, lehrt ihn zu einem gewissen Grade, nur mit weiteren Möglichkeiten die Arbeitsscheue zu pflegen mit allen ihren eigentümlichen Resultaten. Deswegen haben sich ja auch alle ehemaligen ,Internate' einen solchen Ruf verschafft, dass man gezwungen ist, sie zu schließen und Landwirtschaftsschulen einzurichten. “"17 (DVG/KSG 1, 1890)

Die allgemeinbildenden Schulen wurden also für die Einrichtung der Landwirtschaftschulen geopfert, ehemalige Internate in Landwirtschaftsschulen umgewandelt und Ackerland um diese Internate oder im näheren Umkreis gepachtet. Damit verschlechterte sich der Bildungsstandard erheblich. Ein gewisser Zeitanteil, der früher der Allgemein-

17 Weiterhin schlägt der Autor vor, wie die Sesshaftigkeit durch Landwirtschaftsschulen zu erreichen sei: Ein gewisser Prozentsatz aller Geldreichtümer, die in den Kreisen als Spareinlagen aufbewahrt wurden, sollte genommen und in Aktien umgewandelt werden. Die Gewinne aus den Aktien sollten genutzt werden, um Zugtiere und Material zu beschaffen, die dann den Grundstock der Schule bilden. Außerdem sollte ein Teil des Geldes Ackerbauern für einige Tage zinslos geliehen werden. 
bildung vorbehalten war, musste nun der landwirtschaftlichen Betätigung weichen. (Tokseitov 1891)

\section{Bestandsaufnahme: Die Landwirtschaftsschulen im Semirečiekreis}

Im Jahr 1885 wurde vom Generalgouverneur Kolpakovskij veranlasst, in den verschiedenen Gebieten des Semirečie-Kreises Landwirtschaftsschulen einzurichten. (DVG/KSG 21,1888) Sie hatten vor allem ein Ziel: Kasachischen Kindern sollten mit den Mitteln, die in der Steppe vorhanden waren, die einfachsten Handgriffe in der Landwirtschaft sowie Grundwissen in der Allgemeinbildung vermittelt werden. Landwirtschaftsschulen waren dazu gedacht, eine Art Vorbildwirkung auf die Nomaden auszuüben. Man wollte demonstrieren, wie „einfach“ der Zugang zur Landwirtschaft für jeden Nomaden sei. Dabei sollte die Schule mit den örtlichen Möglichkeiten langsam wachsen: Es war anfänglich keine volle Ausrüstung zu erwarten. Das stetige Vergrößern der finanziellen Möglichkeiten sollte durch die eigene Wirtschaftlichkeit erzielt werden. Wie die Landwirtschaft der Nomaden sollten auch die Landwirtschaftsschulen langsam wachsen und erblühen. Die Zielgruppe wurde ganz klar formuliert:

„Je niedriger ein Volk entwickelt ist, desto schwerer fällt es ihm, Erfolge und Resultate in einem längeren Zeitraum zu betrachten. Es will die Resultate jetzt sehen oder wenigstens in der nächsten Zukunft. Einem solchem Volk den Sinn einer Wirtschaft zu vermitteln, die auf den modernen kapitalistischen Grundlagen begründet ist, ist sinnlos, umso mehr es einen Weg kennt, ebensolche Reichtümer zu entwickeln, nämlich mit den Mittel der Viehzucht. [...]

Solange der Nomade noch die Möglichkeit hat, sein Leben mit seinen Viehherde zu bestreiten, sein Leben mit Festen zu verbringen, wird er die Landwirtschaft nicht ins Auge fassen, ja er wird sie mit großem Widerwillen betrachten, wie Dreckarbeit. Die Masse der armen Kasachen jedoch geben das Material ab, eine sesshafte Bevölkerung zu formen.“(DVG/KSG 20,1888)

Die Schulen sollten sich nicht auf den Ackerbau beschränken, sondern - je nach örtlichen Möglichkeiten - auch andere Zweige wie Imkerei, Gartenbau, Plantagenanbau in sich aufnehmen. Auch wurde die Ausbildung in handwerklicher Richtung erwogen, z.B. in der Schmiede, der Walkerei, der Kürschnerei, der Zimmermannskunst. Die einfachsten Fähigkeiten in Arithmetik, Geometrie, Lesen und Schreiben in russischer Sprache, sowie Geographie und anderen für den Ackerbau relevanten Teilen der Naturwissenschaften sollten vermittelt werden. Um auf die örtlichen Gegebenheiten einzugehen, wurden den Schulleitern die größtmöglichen Freiheiten gewährt. Auf Details bezüglich der internen Regelung der Landwirtschaftsschulen wird im Anhang verwiesen.

Im Januar 1895 erschien ein Artikel in der Steppenzeitung, der die Einrichtung von Saatgutvergabestellen innerhalb der Landwirtschaftsschulen empfahl. Es sollte an Landwirtschaft betreibenden Kasachen Saatgut in der Höhe von 2 bis 60 Pud ausgegeben werden. Dieses konnten nach der Ernte bezahlt werden. Spätestens im Oktober des gleichen Jahres war die Schuld zu begleichen. Zur Zurückzahlung sollte das Getreide der Ernte dienen. Bei Missernten wurden Konzessionen gemacht. Da russische Landwirtschaftsexperten bisher nur sehr wenig über geeignetes Saatgut in den Trockengebieten der Steppe wussten, konnten bei Rückgabe gleichzeitig die Qualität der Ernte kontrolliert werden. 
Hiermit sollte ein Ändern der Sorte und eine schrittweise Verbesserung des Saatsortimentes innerhalb der Region erreicht werden. (DVG/KSG 1 1895) In einer späteren Ausgabe der Zeitung ist wiederum die Rede von Sonderverkaufskonzessionen an die Ackerbau betreibende Bevölkerung. Der Verkauf von Pflügen und Saatgut wurde in der Zeitung jetzt erstmals mit einer Ordnung untermauert, die genau über die Regeln der Konzessionen Auskunft gab. Immer wieder liest man in den Bekanntgaben, man solle sich vom Personal der Landwirtschaftsschulen eingehend die Bedienung eines solchen Pfluges zeigen lassen. Ebenso sollten andere Maschinen bestaunt werden, die von den Landwirtschaftsschulen eingesetzt wurden, um sich von der Bequemlichkeit und technischen Überlegenheit der Ackerbaumaschinen zu überzeugen. Auf allen Ebenen sollten die Landwirtschaftsschulen versuchen, so viele Nomaden wie möglich vom Ackerbau zu überzeugen. Mit diesem Schwenk in eine anwendungsorientierte Richtung rückten die Schulen als allgemeine Bildungseinrichtungen immer mehr in den Hintergrund. (DVG/KSG 39, 1895)

\section{Die Probleme in der Etablierung der Landwirtschaftsschulen}

In einem Rechenschaftsbericht der Landwirtschaftschulen wird berichtet, dass die Dreiteilung der Aufgaben in der Schule in den Arbeitsbereich des Agronomen, des Schulleiters und des Kreisbeauftragten ein Problem der Gewaltenteilung mit sich brächte. Jedes der beiden in der Schule angesiedelten Ressorts - das der Allgemeinbildung und das der Agrarbildung - beanspruchte für sich den Hauptplatz. Streitereien seien also vorprogrammiert gewesen. Den Kreisbeauftragten hier als Schlichter einzusetzen wäre eine Utopie, weil er in schulischer Hinsicht keinerlei Autorität darstelle. Es hätte sich herausgestellt, dass sich die Ressorts Agrarbildung und Allgemeinbildung so unvereinbar gegenüber ständen, dass jedweder Schulbetrieb mühselig wäre. Ein weiteres Problem, das gelöst werden müsste, war die Frage der Finanzierung. Es wurden lediglich die Flächen zur Verfügung gestellt, die für den Ackerbau nötig waren. Alles andere sollten die Schulen allein erwirtschaften oder die kasachischen Steuerzahler selbst beisteuern. So gab es natürlich nie ausreichend Geld für einen guten Schulbetrieb. Die Mittel des Ministeriums für Staatseigentum sollten hier Abhilfe schaffen. (DVG/KSG 4, 1890)

Auf der kasachischen Seite waren die Vorbehalte gegen russische Bildungseinrichtungen nicht zu übersehen. Warum sollte ein Kasache in eine Schule gehen und dafür Geld bezahlen, die ihm die Landwirtschaft beibringen wollte, eine Tätigkeit, die weit unten im Sozialprestige der nomadischen Gesellschaft stand? Angesichts dieser Skepsis ist es umso erstaunlicher, dass tatsächlich vereinzelt Kasachen auf diese Schulen gingen. In einem Erlebnisbericht eines Schulabgängers wird über folgende Probleme berichtet: Ein Schüler verließ die Schule, ausgebildet und voller Elan, das erworbene Wissen anzuwenden. Nun fehlte ihm Saatgut, um eine erste Bestellung der Felder zu unternehmen, Felder, die er von seinen Stammesgenossen zur Verfügung gestellt bekommen hatte. Er wendete sich an den Staat und war tatsächlich erfolgreich. Er bekam 25 Pud Weizen geliehen. Als es an das Pflügen der Felder ging und er Pferde anspannen wollte, zerschlugen sie ihm in Panik Pflug und Geschirr, da sie es nicht gewohnt waren, vor landwirtschaftliches Gerät gespannt zu werden. Nach der Reparatur spannte er Ochsen an und diese erwiesen sich als um einiges genügsamer. Die Ernte ließ sich sehen: 600 Pud Weizen. Doch wie sollte der Weizen weiter verarbeitet werden? Die nächste Mühle war etliche Kilometer weit entfernt und verlangte solch einen hohen Mahlpreis, dass sich die Ernte kaum rechnete. Hinzu kam der Aufwand des Transports. Als es im nächsten Jahr wieder ans Ernten ging, ver- 
darb die halbe Ernte, da der Frost Ende Juli vieles zunichte machte. (Nogerbekov/Amre 1894)

In einem Artikel von 1907 regt ein anderer Autor deshalb den Aufbau sogenannter „Erfahrungsfelder“ an. Anhand dieser sollten die klimatischen Bedingungen und andere Fragen der Landwirtschaft (Schädlinge, Bodenverhältnisse usw.) erst einmal richtig erforscht werden. Er bezeichnete die bisherigen Erfahrungen als zufällig und fragmentarisch und zählte eine Vielzahl von Problemen auf, die auftauchten. Die fehlende Fruchtund Bodenkenntnis ließ die Fruchtbarkeit der Böden binnen 3 1/2 Jahren auf Null sinken. Die Böden wurden deswegen oft gewechselt, was zu einer großflächigen Verödung von Böden führte. Der durch die allgemeine Landarmut in Folge der massenhaften Enteignungen erzwungene Übergang kasachischer Viehzüchter von extensiver Weidewirtschaft auf Heuerzeugung tat ein Übriges, auch dadurch nahm die Fruchtbarkeit der Böden ab. Wegen des aufkommenden Schadens sollte nach Meinung des Autors den Kasachen die Heumahd regelrecht untersagt werden. (Škalov 1907) Wenn diese Probleme noch 1907 bestanden, was hatte man dann 20 Jahre zuvor in den Schulen unterrichtet?

Obwohl in Statistiken und Berichten über die Lage der Bildung in der Steppe über die Landwirtschaftsschulen sehr wenig zu finden ist, wird eines deutlich: Die Schulen in der Steppe erreichten nur sehr wenige Leute. Pro Kreis gab es selten mehr als drei Schulen, von denen mindestens zwei in der Stadt angesiedelt waren, nämlich die Grundschulen für Mädchen und Jungen. Meist kam auch noch eine Landwirtschaftsschule hinzu, deren Felder oft in unmittelbarer Stadtnähe oder in der Stadt selbst lagen. Somit konnte nur ein kleiner Teil der nomadischen Bevölkerung erreicht werden. Es waren vor allem russische Kinder in diesen Schulen zu finden. (DVG/KSG 43, 1895) Einer Zeitungsangabe zufolge kamen im Siebenstromland (Semirečie) auf eine Schule 15.762 Einwohner, im AkmolaKreis 25.575 Einwohner. (DVG/KSG 42, 1895) Einer anderen Untersuchung ist zu entnehmen, dass in der Stadt 5.239 Einwohner auf eine Schule kamen, auf dem Lande hingegen 30.089. Nur 0,2\% der Gesamtbevölkerung konnte in diesen ländlichen Schulen jährlich unterrichtet werden. (Kir'jakov 1902, 317f) Die in der Steppenzeitung auftauchenden überschwänglichen Werbeschriften, die die bisherigen Erfolge der eingerichteten Landwirtschaftsschulen aufzeigen sollten, konnten darüber nicht hinwegtäuschen. (Abbas Ajmanov bij Čanov 1890; B-OV 1890) Ein Lagebericht, der die Geschichte der Schulen in den letzten Jahrzehnten umriss, kam zu folgendem Ergebnis: Trotz der langzeitlichen Existenz waren die Schulen nicht auf dem besten Stand. Grund dafür war u.a. ein äußerst dürftiges Budget, welches zum stetigen Verfall der Schulen führte. Eine Revision, die seit 1902 in allen Schulen der Region auf Initiative des Generalgouvernements und der Landwirtschaftsabteilung durchgeführt wurde, ergab, dass sich fast alle Schulen, vor allem die der Landwirtschaft, in einem äußerst bedenklichen Zustand befanden. Die Kommission prüfte nicht nur den Lehrbetrieb, sondern auch die landwirtschaftliche Betätigung. Als Gründe für die Mängel führte sie ein zu kleines Budget, falsche Organisation sowie fehlende Aufsicht und Führung an. Die geringe Entlohnung der Lehrer und die Inflation verunmöglichten eine gute Lehre. Die Schulen waren kaum mit pädagogischem Personal ausgestattet, manchmal fehlte es völlig. Außerdem lagen die Schulen innerhalb der Stadtgebiete, in der Sommerzeit fuhren aber alle aus der Stadt auf die Farmen. So kam es, dass die Schüler weder den Unterricht noch ein Praktikum wahrnehmen konnten, es fehlte an technischem Personal und Lehrern. Die Verlegung der Schulen aufs Land zum dauerhaften Aufenthalt war wegen der Ausgaben für Wirtschafts- und Wohngebäude unmöglich. Die Kosten für anstehende Erneuerungen der Schul- und Wirtschaftsgebäude beliefen sich auf insgesamt 620.000 Rubel für vier Schulen. Dass die wirtschaftlichen Ausgaben (durch ständige Teuerung und wirtschaftliche Misserfolge) mit jedem Jahr 
wuchsen, spiegelte sich im Budget, welches für die schulischen Aufgaben (Lehrmaterial, Pädagogen) vorgesehen war, wider. Es kam bis zur völligen Aufgabe der Lehrtätigkeit (TV 1914, 2). In einer anderen Zeitung ist zu lesen, dass nach fünfjähriger Existenz die Schule in Prschewalsk geschlossen werden musste. Es seien wenige Hoffnungen erfüllt worden, heißt es da. Weitere Gründe für die Schließung wurden nicht genannt. (RZh 1893).

Die Fertigstellung der Orenburg-Taschkent-Linie (1899-1905) und die große Siedlerwelle, die darauf folgte, brachte wesentliche Veränderungen der Schulsituation mit sich. Ein weiterer Artikel über die Lage der Schulen in diesem Zeitraum zeigt folgendes: Die Schulen bekamen eine Finanzspritze, es wurden neue Lehrer eingestellt und Handwerksunterricht angeboten usw. Kurzum, es kommt zu einer Erneuerung des (Landwirtschafts)Schullebens. Die Zahlen von Absolventen und Neuanfängern im Jahre 1909 zeigen, dass es zu einem sprunghaften Anstieg von Neuzugängen kam. ${ }^{18}$ Auf einmal war Geld da, womit der Schulbetrieb wieder in Gang gebracht werden konnten. Land wurde den Absolventen nach erfolgreichem Abschluss jedoch einer Regelung zufolge erst nach 1909 zur Verfügung gestellt. Außerdem wurde festgelegt, dass vier Jahre Bildung in einer Landwirtschaftsschule den Dienst in der Armee aufwogen. Es wurden Stipendien vergeben und der Abschluss von einer solchen Schule aufgewertet. (Dylevskij 1910) Doch damit nicht genug: Es wurden auch sogenannte Schulen für Feldpraktikanten eingeführt, wo spezielle Kenntnisse erworben werden konnten, die z.B. dazu befähigen, selbst in landwirtschaftlichen Einrichtungen zu unterrichten oder qualifizierte Arbeiten in landwirtschaftlichen Betrieben auszuführen (M.A.ev 1916, 2). Es war also eine deutliche Belebung des Schul- und Landwirtschaftssektors zu spüren. Von diesen Veränderungen profitierten jedoch nicht jene Bevölkerungsgruppen, für welche die Schulen ursprünglich bestimmt waren, sondern die russischen Siedler.

\section{Fazit}

Was lehrt dieses Beispiel zaristischer Bildungspolitik bezüglich der Kompatibilität von exogen implementierten Verwaltungsstrukturen auf nomadisch organisierte Gemeinschaften? Hierfür sei noch einmal auf den Anfang zurückgegriffen, auf den Beginn der russischen Verwaltung in der Steppe. Für die ersten Jahrzehnte zwischen 1860 und 1890 lief die Interaktion zwischen beiden Gruppen (kasachischen Nomaden und russischen Verwaltern) erstaunlich gut. Die russischen Kolonialisten beschränkten sich auf die Unterhaltung einer Grenze zu China und überließen den riesigen Steppenraum der nomadischen Selbstverwaltung. So gesehen war in der Tradition der administrativen Toleranz (Katharina II.) die russische Kolonialpolitik auf das Beste geübt im Ignorieren nichtrussischer Belange auf ihrem Staatsterritorium. Bei der ersten großen statistischen Erhebung 1891 wurde aber auch deutlich, welche geringen Kenntnisse man von der Wirtschaftsund Lebensweise der Nomaden hatte. Auch die Debatten in der Bildungspolitik zeigen klar, wie wenig Interesse das russische Imperium über Jahre daran hatte, die Nomaden der Steppe in sein allgemeines Bildungssystem aufzunehmen. Bildung wäre - aus der

1822 Eingeschulten stehen 4 Absolventen in Bischkek gegenüber, 18 Neuzugängen zu 2 Absolventen in der vorher geschlossenen Schule in Prševalsk, 11 Eingeschulte doch keinen Absolventen im Kolpakischen Kreis und 14 Eingeschulte und kein Absolvent in der Gartenbauschule in Verny. 
Sicht der russischen Zentralregierung - von den Nomaden nur dafür genutzt worden, um die Administration mit Beschwerden und die Gerichte mit Klagen zu überhäufen. Dies galt es zu verhindern. An einer gleichberechtigten Interaktion war man weniger interessiert, als an einem klaren Oberhoheits- und Untertanenverhältnis. Aber genau dies fehlte überall dort, wo sich keine russische Hoheit manifestieren konnte: in der Steppe. Im russischen Kolonialreich konnte Macht immer dann gefestigt werden, wenn sie sich auf sesshafte Strukturen stützte. Zwar hatte die russische Politik eine hohe Toleranz gegenüber anderskonfessionellen Gemeinschaften geübt, in dem Moment jedoch, als sie ihre Strukturen in der Steppe etablieren wollte, fehlten ihr die Rezepte, diese Form der „Toleranz" mit nomadischen Strukturen zu verbinden. Als es gerade in der Bildungsfrage um nomadische Partizipation ging, zeigte sich, wie tief die Ignoranz im Staatsdenken und auch in die Staatsraison gegenüber nomadisch lebenden Bevölkerungsgruppen eingepflanzt war. In dessen Folge gingen russische Politiker und deren Ratgeber mit einem nur aus der Tradition der Ignoranz zu verstehenden Überlegenheitsverständnis an die Probleme der Administration der Steppe heran. Sie glaubten, die Angebote, die sie machten, müssten (da sich jedes Volk aus ihrer Sicht „,naturgemäß“ in Richtung der „überlegenen“ Zivilisation entwickeln wollte) dankend von den ,wilden Nomaden“ angenommen werden. Das war - wie der Artikel zu zeigen versuchte - jedoch nicht der Fall.

\section{Bibliographie}

Die hier mit /KSG abgekürzte Quelle ist die Zeitung Dala Viloyati Gazeti Gaziti/Kirgizskaja Stepnaja Gazeta, osoboe pribavlenie $k$ Akmolinskim oblastnym vedomostjam aus Omsk. Sie ist im Mikrofilmarchiv des Seminars für Zentralasienwissenschaften der Humboldt Universität zu Berlin unter der Signatur (VA 2616) einsehbar. Die Abkürzung TV bedeutet Turkestanskie Vedomosti (Taschkent) und ist in eben jenem Mirkofilmarchiv ebenfalls einsehbar. Bei der Abkürzung RZh handelt es sich um die Zeitung Russkaja žisn'.

Abbas Ajmanov bij Čanov (1890) Mestnaja Izvestija, Bajan-aul. In: $D V G / K S G, 15$.

Appasov, S. (1895) O neobxodimosti obrazovanija dlja kirgiz. In: $D V G / K S G, 35$.

Bart'old, V. V. (1963) Kulturnaja Žizn Turkestana, Kapitel VI: Školy. In: Sočinenija II(1), Istorija kul'turoj, Moskau: Nauka.

Bekodžin, X. (1895) K voprosu ob učereždenii škol' v stepi. In: $D V G / K S G, 47,1895$.

Botbaev, M. (1890)Fel'eton. In: $D V G / K S G, 8$.

B-OV., V. (1895) Škola i Žizn‘. In: $D V G / K S G, 32$.

Cabel', L. (1907) Vopros o semleusrtojstv kirgiz na sezde činov pereselenskoj organizatzij turg. ural'sk. rajona. In: Voprosy Kolonisacii, Perjudičeskij sbornik 3, 79100.

Čirkin, G. F. (1907) Zemleotvodnoe delo v kirgizskoj stepi i neobxodimost' zemleustrojstva kirgiz. In: Voprosy Kolonisacii, Perjudičeskij sbornik 3, 59-78.

Čirkin, G. F. (1910) Projektiruemyja Sibirskaja železnyja dorogi i ix kolonizacionnoe značenie. In: Voprosy Kolonisacii. Perjudičeskij sbornik 6, 27-45.

Demko, G. J. (1969) The Russian Colonization of Kazakhstan 1816-1916. Indiana Univ. Publications: Indiana University Publications, Uralic\&Altaic Series:99.

DVG/KSG 20 (1888) Cirkuljar. 
DVG/KSG 21 (1888) Vremenoj ustav kirgizkix sel'skoxozjajstvenyx škol' v oblastjax akmolinskoj i semipalatinskoj.

DVG/KSG 1 (1890) Ob ulušeniji byta džatakov.

DVG/KSG 4 (1890) Ob ustave sel'sko-xozjajstvennyx škol stepnago kraja.

DVG/KSG 33 (1891) ohne Titel [Es handelt sich um einen Artikel über die russischen Schulen für Einheimische, (Anm. d. A.)]

DVG/KSG 10 (1894) O neobxodimosti razvitija zemledelija sredi kirgizkago naselenija.

DVG/KSG 43 (1895) Kakija školy poleznee v stepnom krae.

DVG/KSG 1 (1895) O semennyx skladax dlja kirgiz.

DVG/KSG 19 (1895) Pomošč xlebopaštzam.

DVG/KSG 23 (1895) O neobxodimosti imet' kirgizam svoix spezialno podgotovlenyx učitelej.

DVG/KSG 39 (1895) Petropavlovskij sklad sel'sko-xozjaistvennyx orudij.

DVG/KSG 42 (1895) Po voprosu ob obrasovanii kirgizov.

Dylevskij, A. (1910) Sel'sko-xozjajstvennyx školy semirečija. In: Semirečestkie Oblastnye Vedomosti, 108-110.

Džapakov, K. (1895) Kak obučajut kirgizy svoix detej. In: $D V G / K S G, 8$.

Girs, F./Balljuzek, L./Mejer, L./Protzenko, A. (1960) Vypiska iz objasnitel'noj komissii k projektu položenia ob upravlenii v kazaxskix stepjax, 1867. In: Materialy po istorii politečeskogo stroja Kasaxstana, Bd. 1. Alma-Ata

Hildermeier, M. (1989) Die russische Revolution von 1905 bis 1921. In: Wehler, H. (Hg.) Neue Historische Bibliothek, Frankfurt/M.: edition suhrkamp: 534.

Hopkirk, P. (1994) The great game: The struggle for empire in Central Asia. New York:

Kodansha Globe.

Imankulov, D. (1894) Na pol'su kirgizam. In: $D V G / K S G, 18$.

Khazanov, A. M. (1994) Nomads and the outside world. Madison: University of Wisconsin Press.

Kir'jakov, V. V. (1902) Očerki po istorii pereselenskago dviženija v Sibir (v svjazi s istoriej zaselenija Sibiri). Moskau

Kurmanbaev, A. (1894a) Vzgljad kirgiz na fizičeskij trud. In: $D V G / K S G, 7-8$.

Kurmanbaev, A. (1894b) Kogo kirgizy čitajut xorošim čelovekom? In: $D V G / K S G, 26$.

Lemercier-Quelquejay, C. (1998) Die Kasachen und Kirgisen.In: Fischer Weltgeschichte.

Zentralasien. Bd.16. Frankfurt/M.: Fischer: 152-162.

M.A.ev (1916) Školy polevyx rabočnix praktikantov. In: Turkestanskije Vedomosti,129.

Martin, V. (2001) Law and Custom in the Steppe: The Kazakhs of the Middle Horde and Russian Colonialism in the Nineteenth Century. Richmond: Routledge Curzon.

Masevič M. G. (1960) Materialy po istorii politečeskogo stroja Kasaxstana, Bd 1. AlmaAta

Nogerbekov, A. (1894) Moi zanjatija xlebopašenstvom. In: $D V G / K S G, 49$.

Ohayon, I. (2006) La sedentarisation des Kazakhs dans l'URSS de Staline. Collectivisation et changement social, 1928-1945. Paris: Maisonneuve et Larose.

\section{Abbildungsnachweis}

1. Wikimedia http://upload.wikimedia.org/wikipedia/commons/9/9c/Turkestan_1900-de.svg $(16 / 08 / 2009)$ 
Olaf Günther, Studium der Mittelasienwissenschaften, Geschichte und Gesellschaft Südasiens, Islamwissenschaften und Europäischen Geschichte; Promotion zum

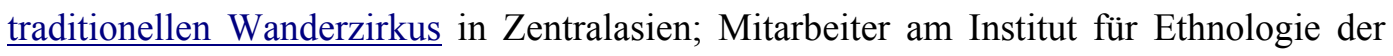
Universität Leipzig, Schwerpunkt: Ethnologie und Kulturgeschichte Zentralasiens; Forschungen zu nomadischen und netzwerkenden Kulturen, besonders von Zigeunergruppen entlang der Seidenstraße; Mitglied des FTF und Redakteur des online Magazins tethys: Central Asia Everyday

E-Mail: olaf.guenther@uni-leipzig.de 